\title{
Numerical Modeling of an Inductively Coupled Plasma Based Remote Source for a Low Damage Etch Back System
}

\author{
Junghoon Joo* \\ Graduate Program of Plasma Convergence Engineering \& Plasma Materials Research Center, \\ Kunsan National University, Kunsan 573-701, Korea \\ (Received July 22, 2014, Revised July 29, 2014, Accepted July 30, 2014)
}

\begin{abstract}
Fluid model based numerical analysis is done to simulate a low damage etch back system for $20 \mathrm{~nm}$ scale semiconductor fabrication. Etch back should be done conformally with very high material selectivity. One possible mechanism is three steps: reactive radical generation, adsorption and thermal desorption. In this study, plasma generation and transport steps are analyzed by a commercial plasma modeling software package, CFD-ACE + . Ar $+\mathrm{CF}_{4}$ ICP was used as a model and the effect of reactive gas inlet position was investigated in 2D and 3D. At 200 300 mTorr of gas pressure, separated gas inlet scheme is analyzed to work well and generated higher density of $\mathrm{F}$ and $\mathrm{F}_{2}$ radicals in the lower chamber region while suppressing ions reach to the wafer by a double layer conducting barrier.
\end{abstract}

Keywords : numerical modeling, inductively coupled plasma, etch back, gas inlet position, CFD-ACE +

\section{Introduction}

Etch back process is getting more interest in fabricating nanometer scale semiconductor devices. Silicon nitride, oxide [1], and poly silicon are main target materials. Main difference between etch back and reactive ion etching (RIE) is ion assistance and etch profile. RIE gives vertical profile through narrow ion incident angle and side wall protection layer formation. Etch back process requires conformality which is not attainable in RIE. Atomic layer etching would be a solution to etch back process. It has very slow etching rate which may be unacceptable in current device fabrication requirements. One prerequisite of this etch back process is no ion damage. A re- mote plasma source [2-4] would be a candidate where volume or surface recombination of ions occurs in appropriate process pressures. To keep high etch rate and throughput of wafers, some thickness of reaction layer should be formed in exposure to plasma. In second step, the wafer is transferred to a thermal desorption chamber and processed [5]. The intermediate reaction byproduct, e.g. $\left(\mathrm{NH}_{4}\right)_{2} \mathrm{SiF}_{6}$ in plasma oxide cleaning process by $\mathrm{H}_{2}, \mathrm{~N}_{2}, \mathrm{NF}_{3}$ is unstable product with very low vapor pressure at room temperature. Similar case can be found in literature [6]. It should be heated higher than $100^{\circ} \mathrm{C}$ to desorb. HF may play an important role in this process. Some manufacturers are using vapor process and others are using plasma as a source of $\mathrm{F} . \mathrm{CF}_{\mathrm{x}}$ gases are mostly

* [E-mail]jhjoo@kunsan.ac.kr 
used in oxide and nitride etching processes which have hard C layer on Si surface to give vertical profile as well as high selectivity of oxide/nitride to silicon. For etch back processes, a plasma source should have high density of radicals with low ions. In this study, 2D and 3D numerical models are used to investigate how the source gas is getting dissociated and ions are eliminated. Microwave plasma based chemical downstream etch system modeling was reported by Meeks [7].

\section{Numerical Model Setup}

Plasma fluid equations are valid in moderate gas pressure (higher than mTorr). Inductively coupled plasmas (ICP) are well represented by this fluid model. $\mathrm{CFD}-\mathrm{ACE}+$ (ESI corp) is a commercial fluid code based on multiphysics modules. Plasma modeling includes fluid, heat, chemistry, electromagnetic and electron temperature modules. Detailed governing equations and validation studies are published elsewhere. 2D and 3D models are available in CFD-ACE+. 2D axisymmetric models are much faster than 3D which would take several weeks to get fully converged. Gridding scheme can have either structured or unstructured meshes. In order to investigate the effects of reactive gas inlet position on the discharge chemistry, we used further compact model using quasineutrality conditions where Poisson equation is not solved and reducing computational time. It is very useful in 3D models. Inductively coupled antenna is generally spiral wound around a dielectric tube made of alumina, $\mathrm{Al}_{2} \mathrm{O}_{3}$ or quartz depending on the reactive gases. 2D model has 25,000 rectangular cells and 3D model has 550,000 hexagonal cells. RF antenna in ICP model generally invokes complex geometry grid which would make difficulty in constructing all structural grids. Interpolation scheme, named filament model in $\mathrm{CFD}-\mathrm{ACE}+$, is used and saves large

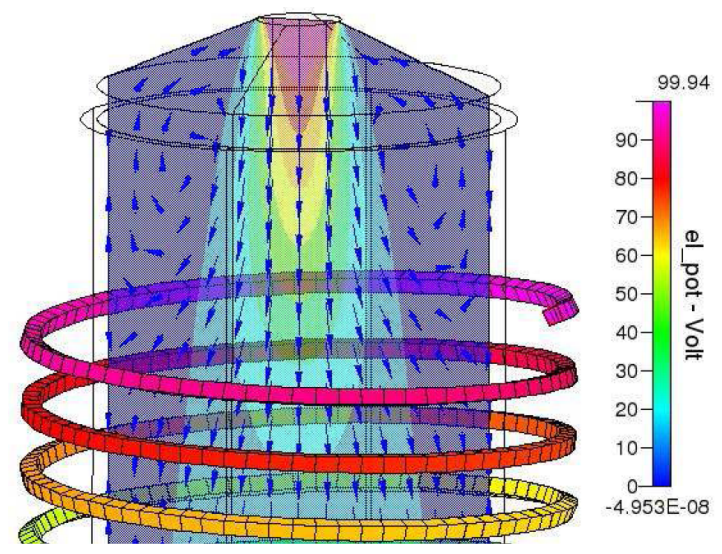

Figure 1. A filament model represents an RF antenna saving complex unstructured gridding.

number of meshes as shown in Fig. 1. A 3D model required a month of CPU time to achieve a converged solution. Parallel processing was not efficient in a filament model, because decomposed zone boundary should not pass through a filament. One could bypass this limitation by realizing an ICP antenna and the volume around by tetrahedral meshes. But a doubled number of cells would decrease the advantage of a parallel processing.

\section{Simulation Results and Discussion}

\section{Gas flow characteristics}

Longitudinal cylinder type ICP sources have gas flow uniformity issues. A gas distribution plate (GDP) may be a solution. In Fig. 1, small area central inlet supplies gases and inherently have a problem of vortex generation between central gas flow region and side wall corner region. Process uniformity of reactive gas plasmas is strongly dependent on neutral gas supply. In our model, the diameter of ICP tube is around $100 \mathrm{~mm}$. Power absorption depth of ICP is decreasing as the plasma density increases. If it goes below the gas flow boundary layer thickness, gases would be overheated. Dielectric materials are used in 
confining ICPs and are affecting surface reactions: ionic recombination, secondary electron emission and sputtering. Plasma potential is affected by capacitive coupling of the ICP antenna and the plasma. Faraday shields are used to keep this potential low. Side effects are lowered rf power coupling efficiency. In high power applications of ICP, e.g. thermal plasma torches, dielectric walls are eroded easily. Low pressure and high power applications like remote plasma cleaning would include appropriate cooling of the dielectric tubes, especially when reactive gases are mixed.

\section{Electron temperature and density distribution}

Plasma profile is usually interpreted as the electron density profile and is determined by ion generation and loss to surfaces in low pressure gas discharges. In our model, slightly high gas pressure is used, 300 mTorr. At this pressure, neutral - neutral collision mean free path is $0.17 \mathrm{~mm}$. Power absorption model for ICP includes Ohmic heating and non-local processes. Our model is using only Ohmic heating as explained in references [8-10]. Fig. 2 is showing the calculated skin depth is less than $10 \mathrm{~mm}$ at this simulated power absorption. Electron temperature was calculated with Maxwellian distribution assumption showing $1.5 \mathrm{eV}-1.3 \mathrm{eV}$ around ICP antenna region in Fig. 3. Electron density shows center high profile (peak $8 \times 10^{18} \mathrm{\#} / \mathrm{m}^{3}$ ) dominated by surface recombination loss reactions in Fig. 4. Electron transport properties, e.g. the diffusion coefficient, would determine the electron density profile in the lower region. Electron-neutral collision frequency is a major factor and the kind of gases, too. In 2D modeling, Ar was used and in $3 \mathrm{D}$ ones, $\mathrm{Ar}+\mathrm{CF}_{4}$ was used either

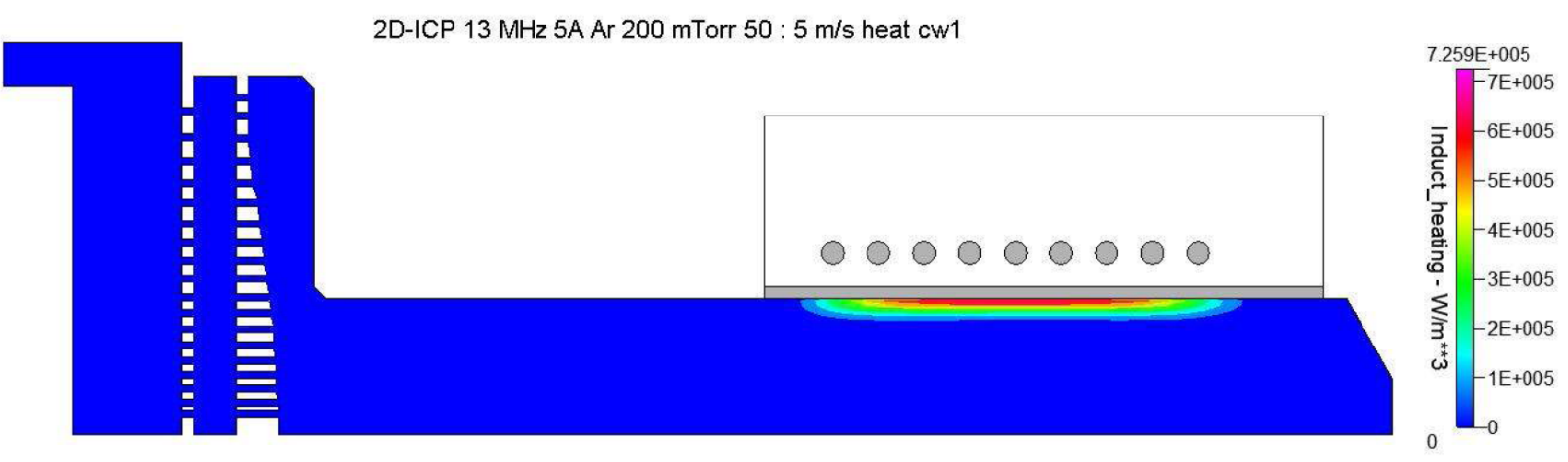

Figure 2. Inductive power absorption profile in 2D model.

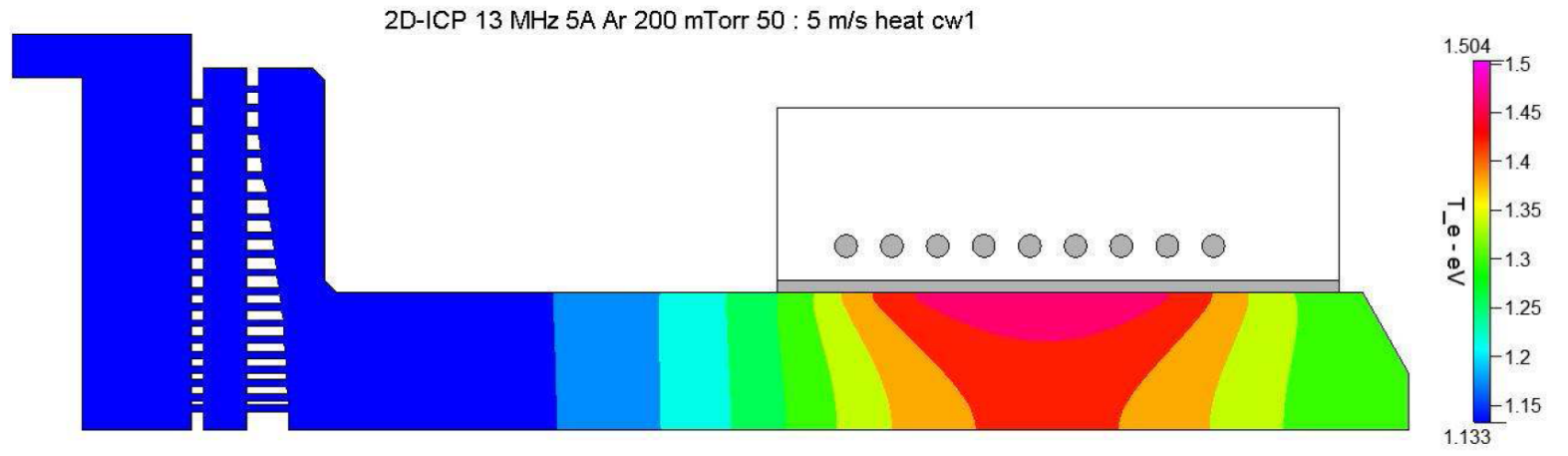

Figure 3. Electron temperature profile in 2D model. 


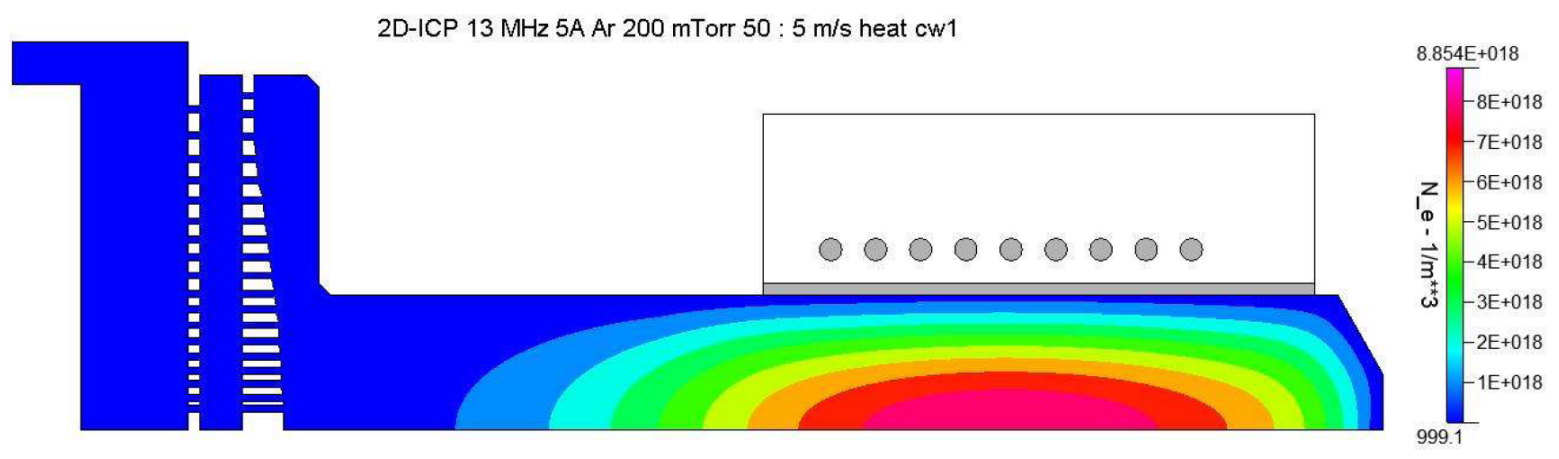

Figure 4. Electron density profile in 2D model.

(a)

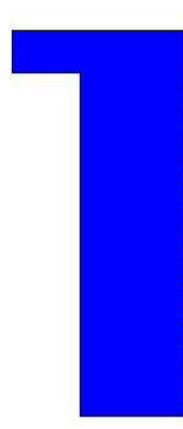

(b)

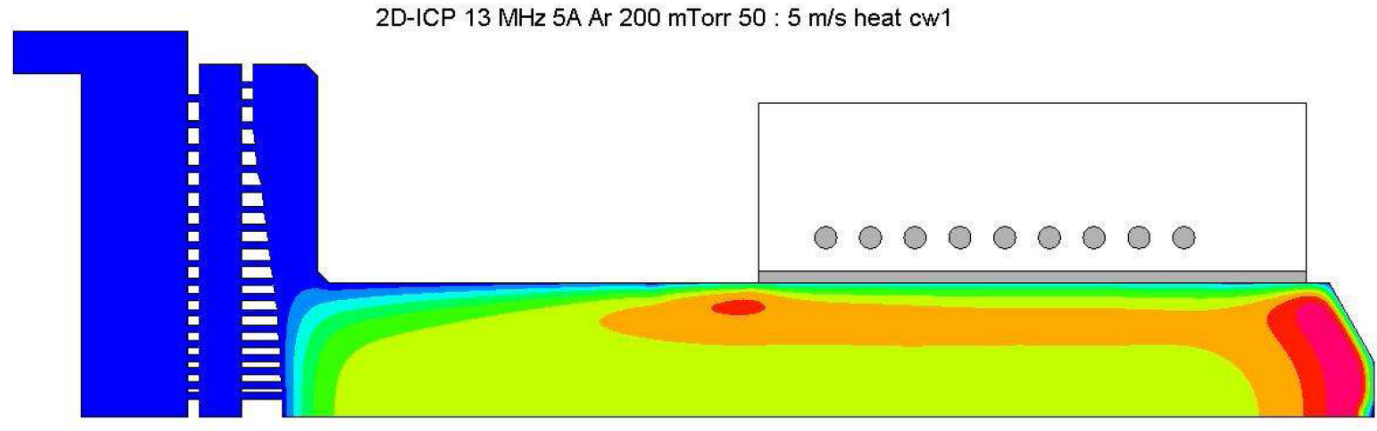

2D-ICP $13 \mathrm{MHz} 5$ A Ar $200 \mathrm{mTorr} 50: 5 \mathrm{~m} / \mathrm{s}$ heat $\mathrm{cw} 1$

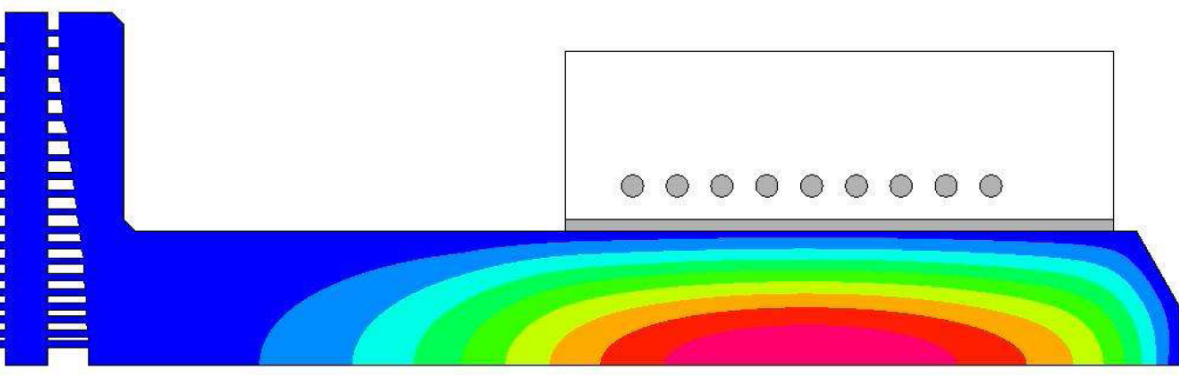

2D-ICP $13 \mathrm{MHz} 5$ A Ar $200 \mathrm{mTorr} 50: 5 \mathrm{~m} / \mathrm{s}$ heat $\mathrm{cw} 1$

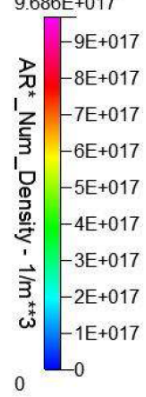

Figure 5. (a) Ar ion (b) Ar metastable number density profile in 2D model.

in mixed form or in separated inlet conditions. One of the purposes of separated inlets is to control the density of $\mathrm{F}$ radical and related products, e.g. $\mathrm{F}_{2}$. As the neutral diffusion is a strong function of gas temperature, heat equations are solve d [11]. Electron elastic collision, ion Ohmic and ion surface heating is considered in generalized heat transfer equation. At low pressure discharge as 1 10 mTorr, fluid model should include ion momentum equation to drift diffusion approximation for better accuracy. Under quasineutrality approximation, sheath is analyzed by a model not by resolving with Poisson equation.

\section{Heavy particle number density distribution}

Ar ion and metastable number density is shown in Fig. 5a and 5b. Ar ion profile is similar to electron density profile. Ar metastable shows much different spatial distribution. Number density of $\mathrm{Ar}^{*}$ is $1 / 10$ of Ar+ in most of regions. Ar* has two peak areas: top gas inlet and bot side inlet. Gas inlet velocity was set to 50 $\mathrm{m} / \mathrm{s}: 5 \mathrm{~m} / \mathrm{s}$ each. 2D axi-symmetric model describes radial gas inlet at reduced velocity by $1 / 2 \pi$ radian. Electron impact excitation of ground state Ar to $\mathrm{Ar}^{*}$ requires $11.5 \mathrm{eV}$. Ar* density has peaks where elec- 
tron temperature is relatively low but high density of Ar ground state neutrals and electrons. It means Penning reactions (excitation, dissociation and ionization) could have much different distribution from direct ionization profile. In designing of complex reactive gas reactor, it should be considered seriously as well as rf power absorption profile controlled by rf electrode or antenna shape.

\section{Gas temperature profile}

Calculation of neutral gas temperature distribution includes ion impact surface heating. Ambipolar potential profile is shown in Fig. 6. Maximum potential is around $30 \mathrm{~V}$ and lower than $10 \mathrm{~V}$ around the wafer. Double layered baffle may play a role of ion removal by surface recombination. For radicals, e.g. F and $\mathrm{CF}_{\mathrm{x}}\left(\mathrm{CF}, \mathrm{CF}_{2}\right.$ and $\left.\mathrm{CF}_{3}\right)$, surfaces would be very sensitive sites for recombination and deposition as a func- tion of surface temperature and surface site state, e.g. damage, contamination and electric potential (surface charge state). In most of numerical modeling, chamber inner surfaces are considered to be $100 \%$ recombination site of ions. But radicals have much lower probability of recombination or de-excitation to ground levels. At 200 300 mTorr of pressures, gas phase collisional recombination would be a non-trivial portion of $\mathrm{F}$ induced molecular species, e.g. $F_{2}$ and HF. Non UHV chambers have fairly large amount of water and it is dissociated into $\mathrm{H}_{2}, \mathrm{H}$ and $\mathrm{OH}$. Even with $\mathrm{Ar}+\mathrm{CF}_{4}$ discharge, $\mathrm{HF}$ could be produced via collisions between $\mathrm{F}$ and dissociated species from water. QMS (quadrupole mass spectrometer) study would show the effect of lingering water effects on the plasma processing of wafers with a load/lock transfer.

Calculated temperature profile is shown in Fig. 7. Maximum temperature is $894^{\circ} \mathrm{C}$ at the center line in

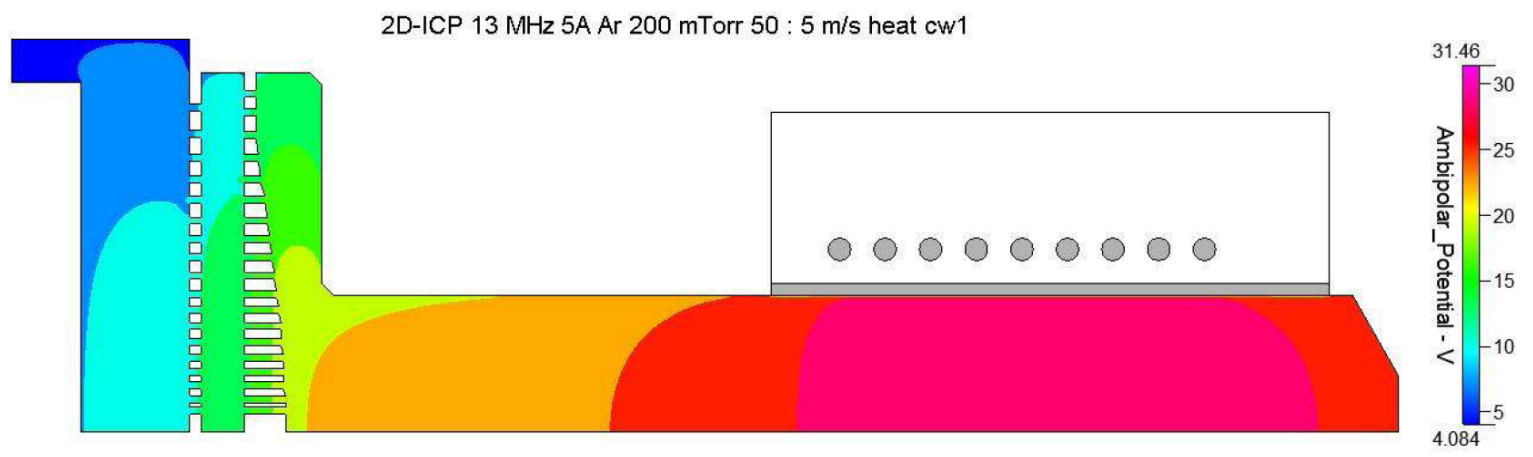

Figure 6. Ambipolar potential profile in 2D model.

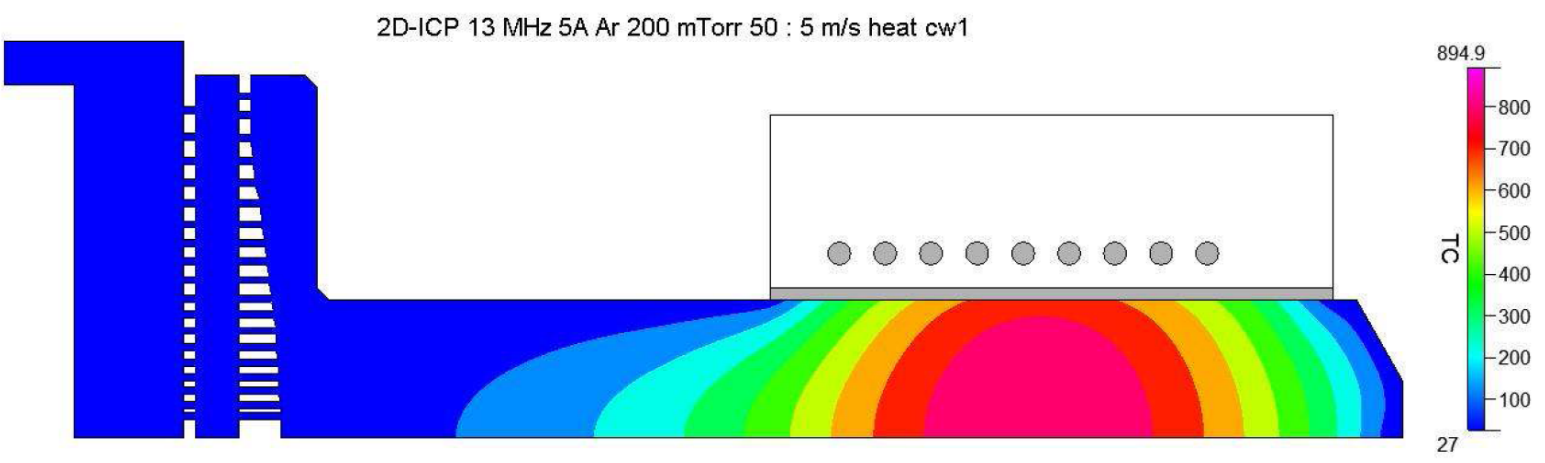

Figure 7. Neutral gas temperature profile in 2D model. 


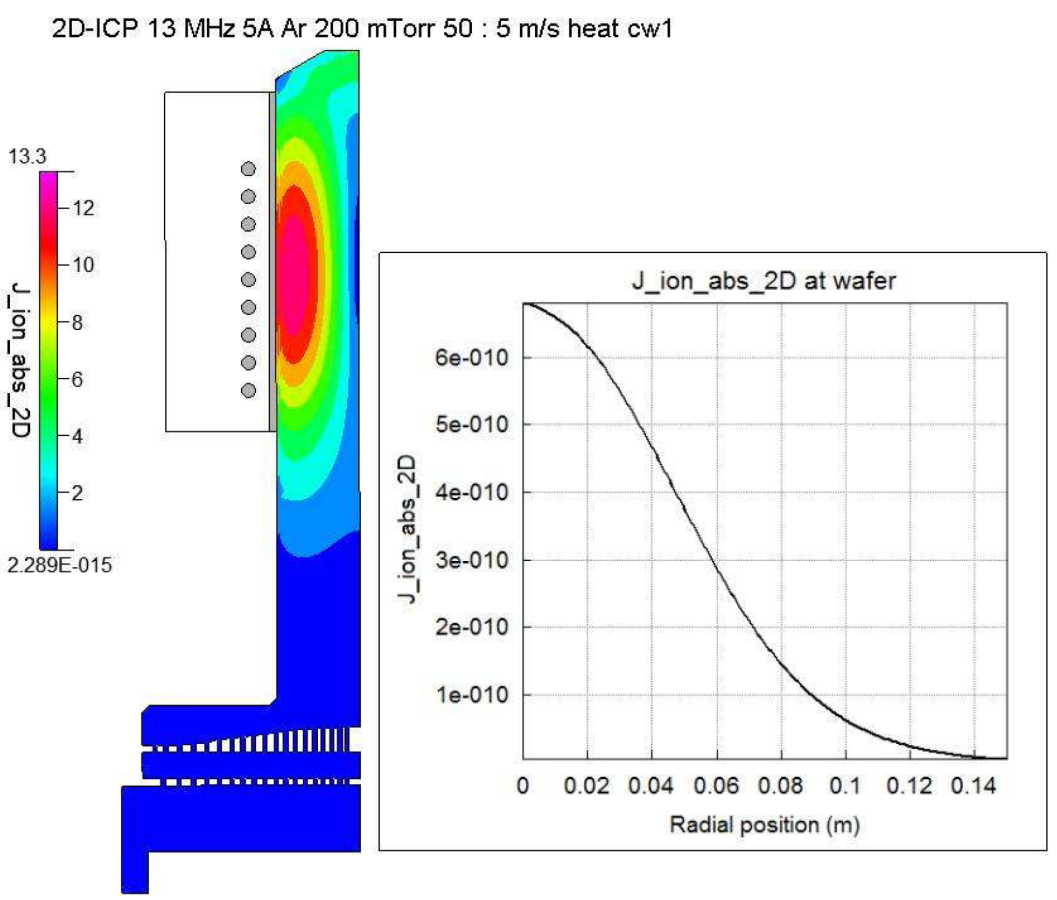

Figure 8. Ion flux density profile at wafer as a function of radial position in $2 \mathrm{D}$ model.

the ICP region. At the downstream region, it is lower than $100^{\circ} \mathrm{C}$. Chamber walls are assumed to be $25^{\circ} \mathrm{C}$. Many dry etcher manufacturers are using temperature controlled chamber walls to enhance processing uniformity and to suppress polymer deposition which could invoke particle generation when it gets too thick and over-stressed. Thermal radiation was not considered in this modeling. If the neutral gas temperature exceeds $1,000^{\circ} \mathrm{C}$, thermal dissociation of reactants should be considered in modeling. $\mathrm{NF}_{3}$ would be a case. At the wafer level, ions are almost removed as shown in Fig. 8. In the center of ICP region, the ion saturation current density is around $10 \mathrm{~A} / \mathrm{m}^{2}$.

\section{Effects of gas inlet position}

Reactive gases are introduced into the chamber via some type of gas distributors. A shower head style gas distribution plate (GDP) is a good example. Couett flow is the underlying principle of uniform gas ejection from thousands of gas holes in GDP, even supplied with one or two quarter inch diameter of gas tubes. Downstream reactor design may have very long

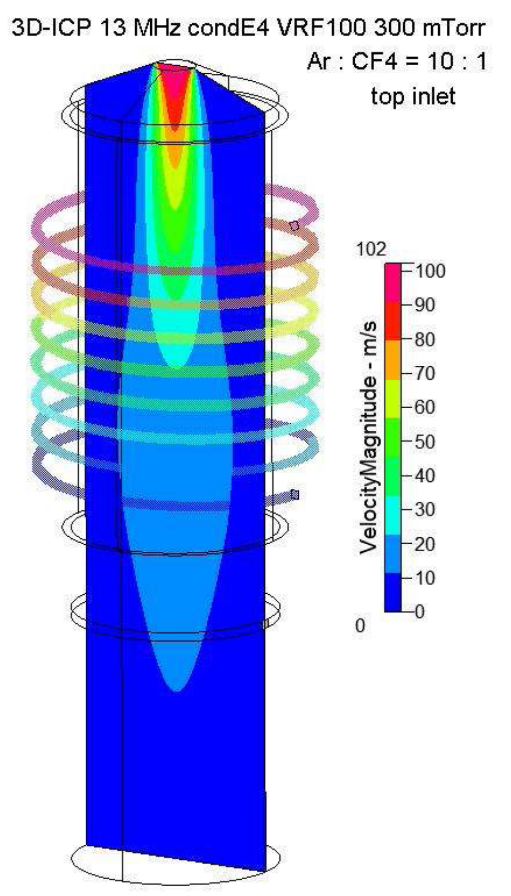

Figure 9. Gas velocity profile at $Y$-cut plane in 3D model with top inlet.

drift region between a plasma generation region and a reaction chamber. So called hot region may not be the right position for gas introduction. Over dissociation would harm the selectivity of the etch back 
3D-ICP $13 \mathrm{MHz}$ condE4 VRF50 Ar $300 \mathrm{mTorr}$ $10: 100 \mathrm{~m} / \mathrm{s}$ heat



Figure 10. Isosurface of $1 \mathrm{~m} / \mathrm{s}$ velocity filled by laminar viscosity. process. In Fig. 9, the gas velocity profile of a 3D model is shown and have very slow flow around the dielectric tube. Central region has high velocity of $100 \mathrm{~m} / \mathrm{s}$ at the vicinity of top inlet. But it is slowed down at ICP region. Small size inlet than the diameter of the dielectric tube will have some stagnant region around the inlet boundary as shown in Fig. 1. After traveling some distance, it will develop to a uniform laminar flow. Right position for installing ICP antenna will be after the developing region from the inlet boundary. In Fig. 9, the 7th turn of the ICP antenna has the widest developed flow in the radial direction. So the improved version of this ICP remote source would set the center to this position. In Fig. 10 , the iso surface having $1 \mathrm{~m} / \mathrm{s}$ velocity is shown and colored by laminar viscosity. It is showing the effect of bottom side inlet to the gas flow dynamics. For the results in Fig. 12 and after, Ar is introduced from the top inlet and $\mathrm{CF}_{4}$ is coming from the bottom side inlet. 3D model represents more accurate gas inlet (a)

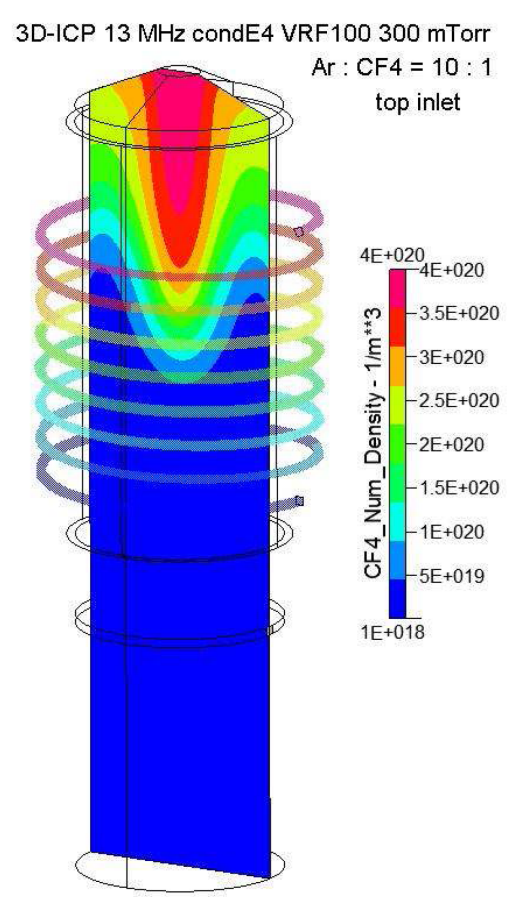

(b)

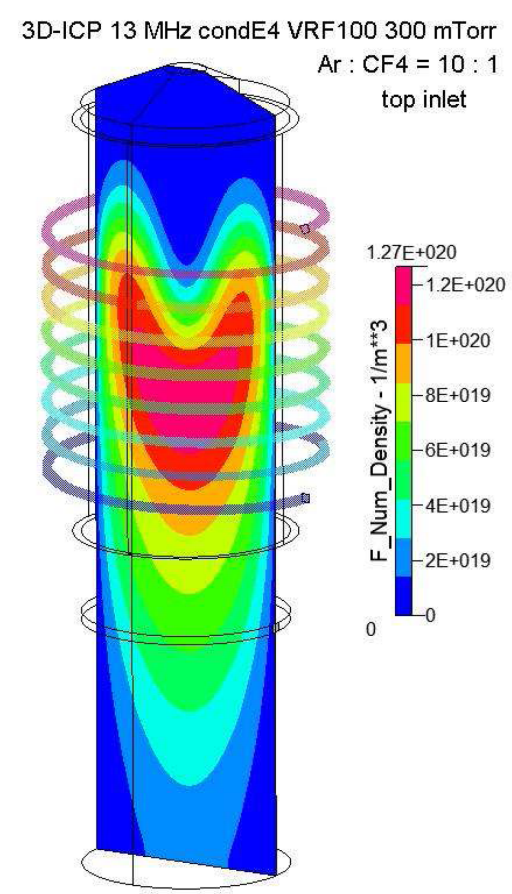

(c)

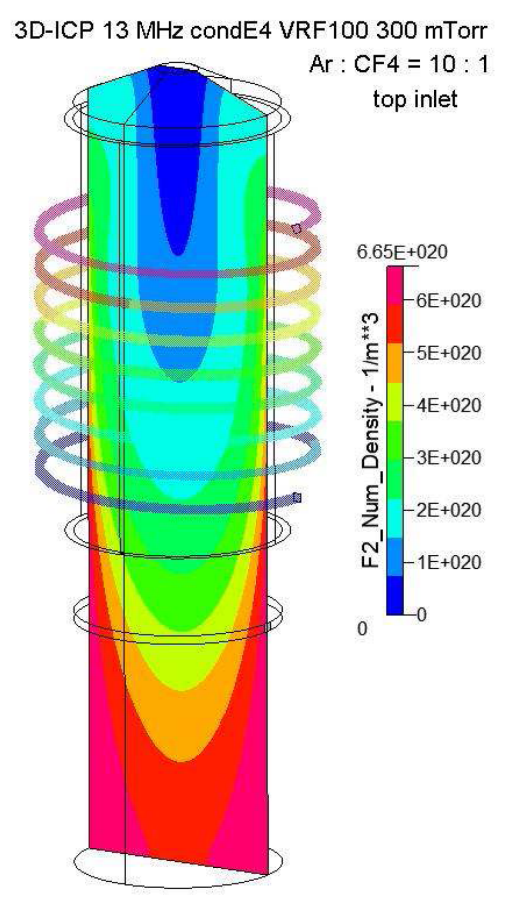

Figure 11. (a) $\mathrm{CF}_{4}$,(b) $\mathrm{F}$, (c) $\mathrm{F}_{2}$ number density profile in 3D model with top inlet. 


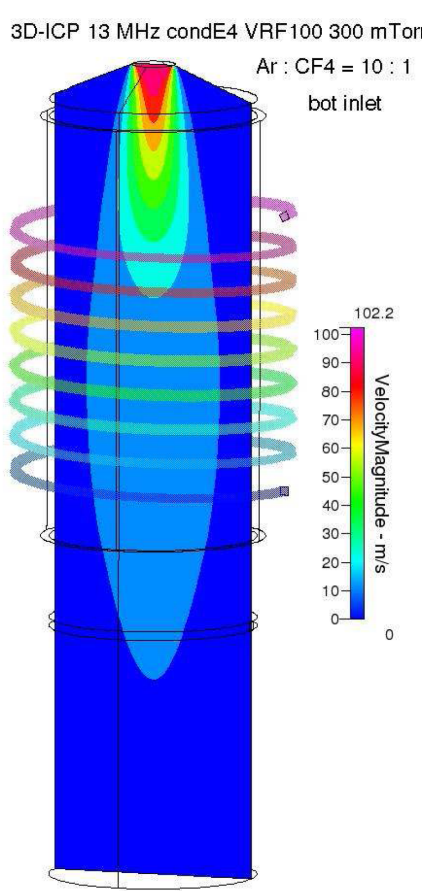

Figure 12. Gas velocity profile at $Y$-cut plane in 3D model with separated inlets

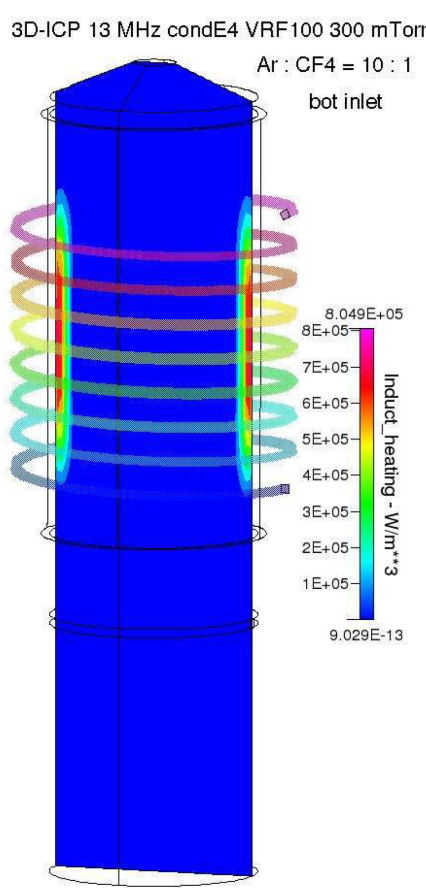

Figure 13. Inductive power absorption profile in 3D model with separated inlets
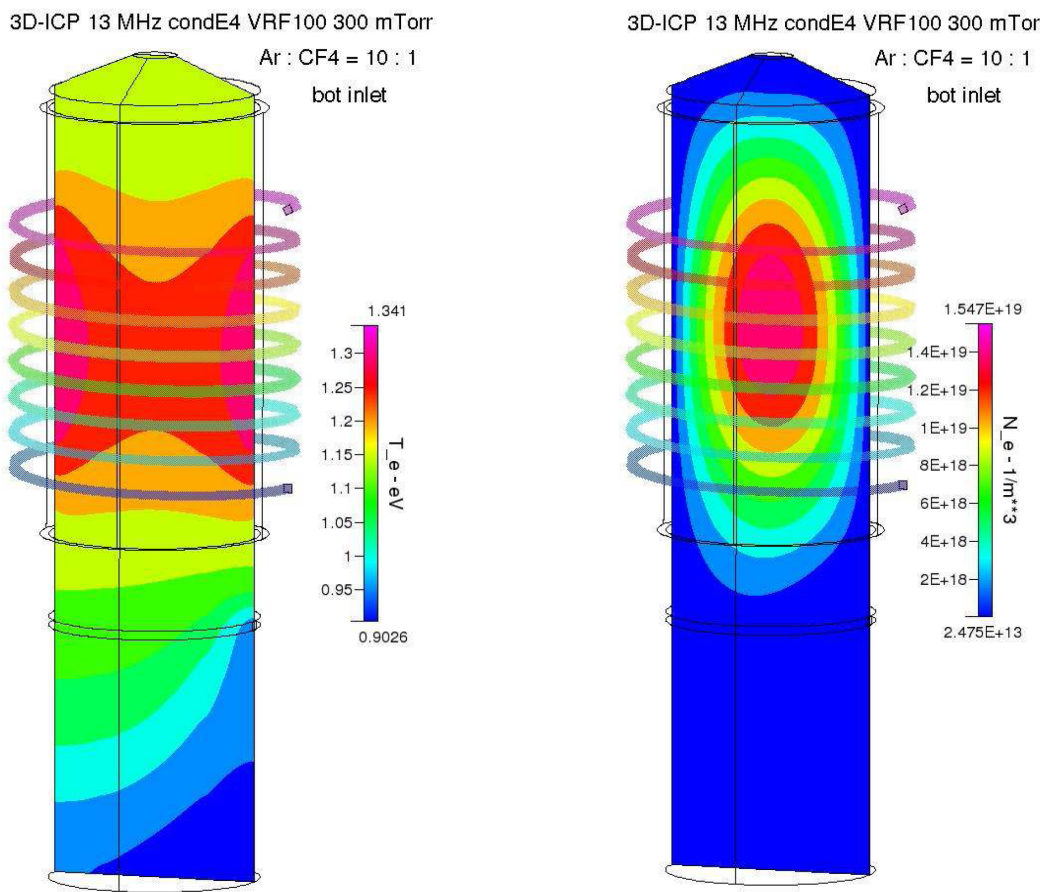

Figure 14. (a) Electron temperature, (b) density profile in $3 D$ model with separated inlets.

velocity than the $2 \mathrm{D}$ axi-symmetric model. For simple numerical gridding, the inlet area is approximated to a rectangular region not a circular cross section of a tube. In Fig. 11, $\mathrm{CF}_{4}, \mathrm{~F}$ and $\mathrm{F}_{2}$ number density are shown in (a), (b) and (c). $F_{2}$ generation path is included only by the surface recombination of two $\mathrm{F}$ radicals. If a volume recombination were considered, $\mathrm{F}_{2}$ would have higher concentration at the lower part 
(a)

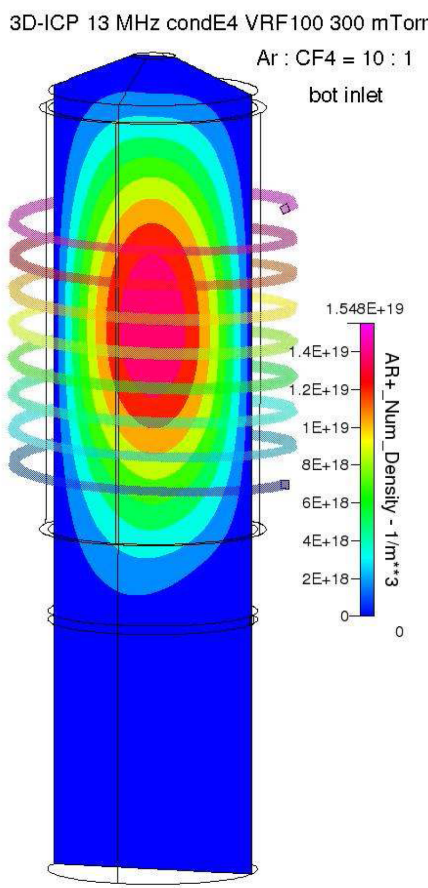

(d)

3D-ICP $13 \mathrm{MHz}$ condE4 VRF100 $300 \mathrm{mTorr}$

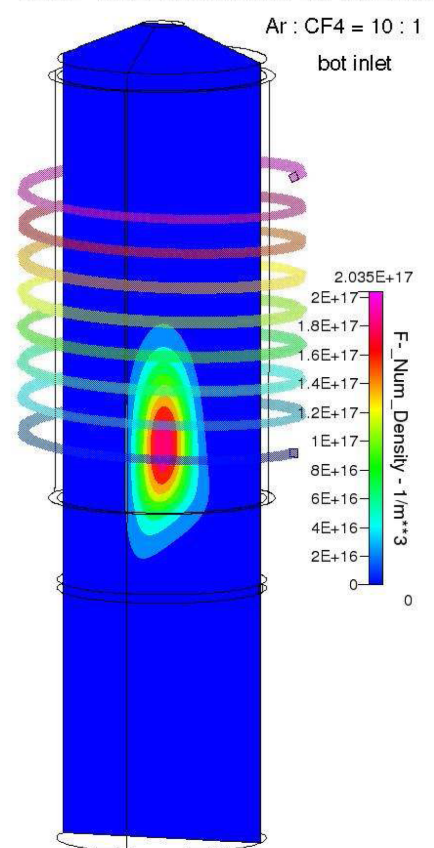

(b)

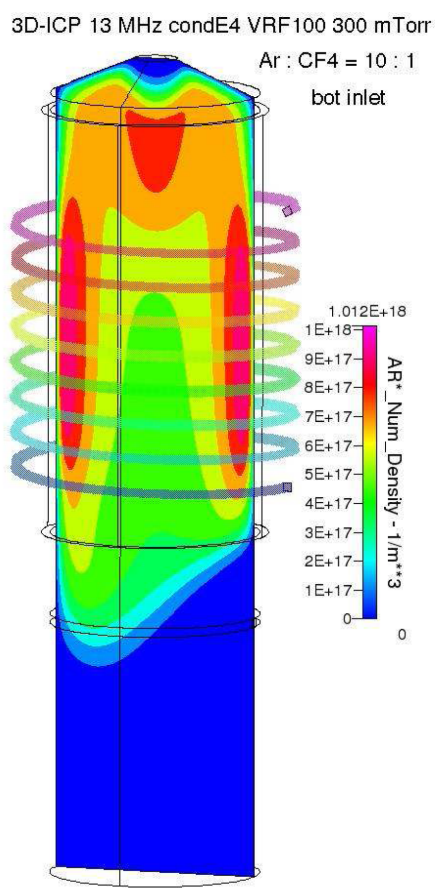

(e)

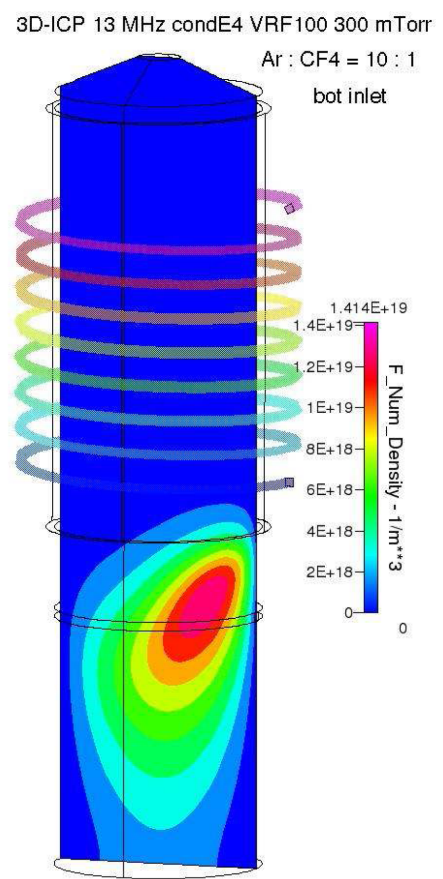

(c)

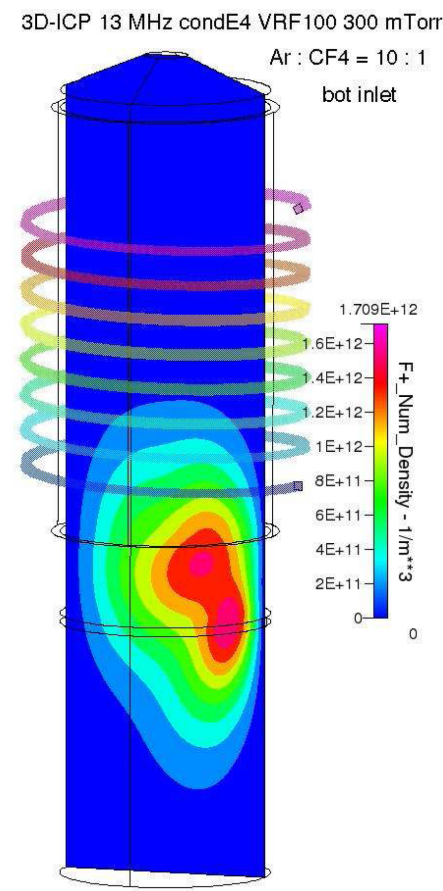

(f)

3D-ICP $13 \mathrm{MHz}$ condE4 VRF $100300 \mathrm{mTorr}$

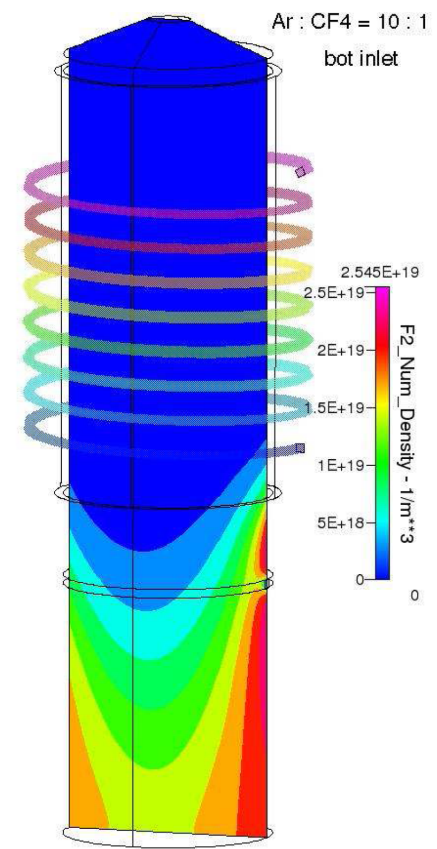

Figure 15. (a) $\mathrm{Ar}^{+}$,(b) Ar metastable, (c) $\mathrm{F}^{+}$, (d) $\mathrm{F}^{-}$, (e) $\mathrm{F}$, (f) $\mathrm{F}_{2}$ number density profile in 3D model with separated inlets.

of ICP region where dissociated $\mathrm{F}$ radicals begin to collide. Gas velocity distribution in Fig. 12 is not much different from top inlet case of Fig. 1. It is because the bottom side inlet velocity is $10 \mathrm{~m} / \mathrm{s}$ and the inlet area is smaller than the top inlet. Inductive power absorption profile in Fig. 13, the electron temperature and density profile in Fig. 14 (a) and (b) are very similar to that of top inlet case. Electron tem- 
perature is slightly lowered in the bottom side inlet region where $\mathrm{CF}_{4}$ is coming in. Heavy particle profiles are shown in Fig. 15. Ar metastable profile in (b) is different from the top inlet case taking after inductive power absorption profile. Calculated $\mathrm{F}^{+}$number density is in $10^{12} \# / \mathrm{m}^{3}$ and $\mathrm{F}^{-}$is in $10^{17} \# / \mathrm{m}^{3}$. F radical number density is also $10^{19} \# / \mathrm{m}^{3}$. Bottom side inlet case shows reduced F positive ion number density in the downstream region and dominant $\mathrm{F}$ negative ion and neutral density. This is an advantage of a separated gas inlet system for low damage etch back where ions should be suppressed and only radical adsorption be used. The role of negative ions, whose number density is a few percent of $\mathrm{F}$ radical density, should be investigated further. Since the etch back system would not use any rf bias, the plasma potential should be kept low to suppress ion induced damage at the wafer. If rf bias were used, the plasma potential would go into positive period and the negative ions would bombard the wafer surface with enough kinetic energy to create damages. RF pulsing may be another method to make use of negative ions in etching.

\section{Conclusion}

Numerical simulation of an ICP based remote plasma system is successfully done both in $2 \mathrm{D}$ and $3 \mathrm{D}$ by using fluid model with quasi-neutrality approximated drift diffusion approach. For low damage etch back system, radical based processing is necessary. A double layered conductive barrier was effective in suppressing ions to a wafer. In a model of $\mathrm{Ar}+\mathrm{CF}_{4}$, effects of reactive gas inlet position was investigated. A bottom side inlet model showed $\mathrm{F}$ and $\mathrm{F}_{2}$ radical dominant density distribution in the lower chamber region compared with a top inlet case. F negative ions was calculated to be a dominant ionic species, but it was very much localized at the center of the discharge region.

\section{Acknowledgement}

This work was supported by WC300 research project by Korean government.

\section{References}

[1] S. K. Kwon, D. H. Kim, and J. T. Baek, J. Cry. Growth, 198/199, 1039 (1999).

[2] Junghoon Joo, Proceedings of International Conference on Microelectronics and Plasma Technologies 2008, Jeju, 531.

[3] Yeonguk Kim, Wonkyun Yang, and Junghoon Joo, J. Kor. Inst. Surf. Eng., 41, 134 (2008).

[4] K. H. Lee, W. K. Lee, Y. K. Lee, S. W. Jo, and C. W. Chung, Sae Muli, 55, 28 (2007).

[5] PSK inc, Korean patent No. 10-2011-0110517.

[6] H. Xian-wei, L. Lin, G. Bing-liang, S. Zhong-ning et. al, Trans. Nonferrous Met. Soc. China, 21, 2087 (2011).

[7] E. Meeks, R. S. Larson, S. R. Vossen, and J. W. Shon, J. Electrochem. Soc., 144, 357 (1997).

[8] Junghoon Joo, J. Kor. Inst. Surf. Eng. 45, 174 (2012).

[9] Junghoon Joo, Thin Solid Films, 519, 6892 (2011).

[10] Sangwon Lee, J. Kor. Vac. Soc, 18, 176 (2009).

[11] CFD-ACE+ user manual module II, 197, 2013. 